\title{
Ecological and hydrophysical research of impact the vertical turbulent exchange coefficient on the concentration of dissolved oxygen in the bottom layer of shallow water
}

\author{
Alexander Sukhinov ${ }^{1, *}$, Alla Nikitina $^{2}$, Yulia Belova $^{1}$ and Tatyana Bednaya ${ }^{1}$ \\ ${ }^{1}$ Don State Technical University, Rostov, Russia \\ ${ }^{2}$ Southern Federal University Taganrog, Russia
}

\begin{abstract}
The paper presents the results of hydrochemical observations obtained during the expedition on the scientific-research vessel "Deneb" in the Eastern part of the Azov Sea and the Taganrog Bay in July 2017. In the ecological and hydrophysical researches we have found abnormally low levels of dissolved oxygen in the bottom layer of the Azov Sea. Among the various approximations of the vertical turbulent exchange coefficient, the algebraic subgrid models are the most applicable. These models are based on the determination of turbulent flows as averaged multiplication of deviations over space or time (correlation) of the velocity components of the currents and a transferring physical quantity. The numerical experiment for determination of vertical turbulent exchange coefficient for some points of the Azov Sea was performed. Direct dependence between values of the vertical turbulent exchange coefficient and the concentration of dissolved oxygen in the bottom layer was obtained.
\end{abstract}

\section{Introduction}

Human impact on nature along with the abnormal climate changes lead to the violation of the natural functioning of the hydro-ecosystems, eutrophication, decreasing of biological diversity. Pollutants accumulated in bottom sediments. During the summer, sediments are heated, and the sludge microorganisms release substances that immediately cause the release of hydrogen sulfide. The decomposition of huge amounts of organic matter in a short time period leads to a significant reduction in the concentration of dissolved oxygen in the water, causing fish kills and, as a result, leads to water quality deterioration. Among all hydrochemical parameters influencing on the biological productivity of the Azov Sea, the dissolved oxygen has a special attention as the source of the respiration of aquatic organisms and the factor determining the speed and completeness mineralization of organic matter in the water. A special feature of the oxygen regime of the Azov Sea is in its instability, which is defined by permanent and seasonal factors: the absorption of atmospheric oxygen by the surface water layer, the uneven inflow of rivers and Black Sea waters, the development and decay of organisms, circulation processes determining the possibility of oxygen penetration in the bottom layers, and other processes. The concentration of dissolved oxygen in sea water depends on several factors, the main ones are: the gas exchange between water and atmosphere; the intensity of the photosynthesis process; the process by which bacterial and chemical oxidation metabolites of marine organisms, remains of dead organisms of plant and animal origin; the intensity of convective mixing and the flow of oxygen-depleted waters; water advection of masses, biochemical oxygen demand of bottom sediments [1].

In summer under conditions of density stratification occurs enhanced reduction of oxygen concentration in bottom horizons of the Azov Sea (in some areas up to $60 \%$ ). The leading factors of formation of the oxygen regime in surface waters are primarily production processes, in the bottom - the biochemical decomposition of bottom sediments. The oxygen in the process of invasion, penetrates into the underlying layers through turbulent exchange, mainly vertical turbulent diffusion in stratified waters [2-6].

The aim of this paper is verification the influence of hydrodynamic conditions on the change of dissolved oxygen concentration.

\section{Expidition route, equipment}

The scientific-research vessel "Deneb" (SRV "Deneb") of the Southern Scientific Center of the Russian Academy of Science RAS (SSC RAS) was used to performed scientific researches in the Azov Sea.

The researches were performed at 17 stations in the Eastern part of the Azov Sea (Fig.1).

\footnotetext{
* Corresponding author: sukhinov@gmail.com
} 


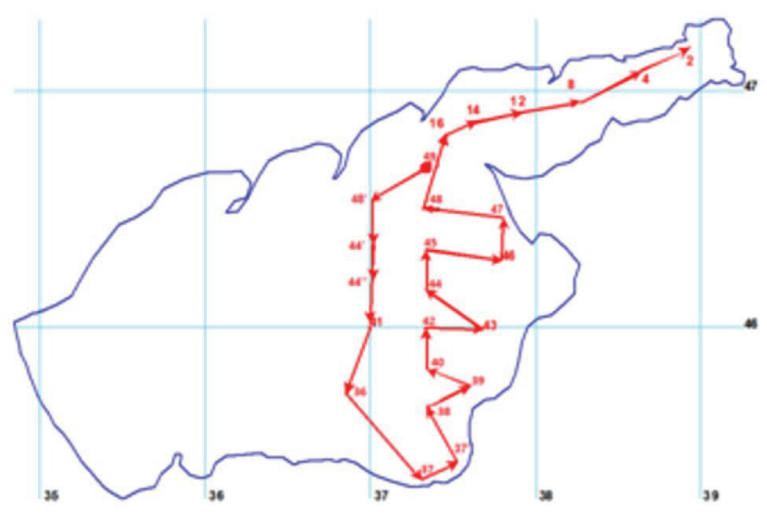

a)

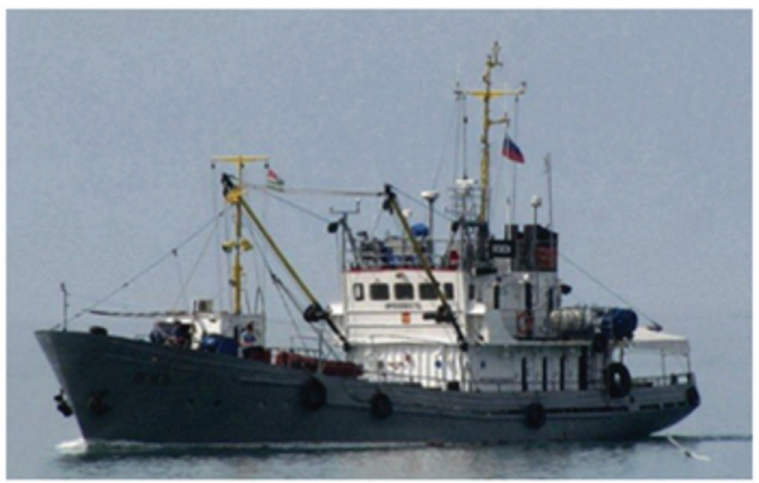

b)

Fig. 1. Scientific researches in the Azov Sea (July 2017): a) the expedition route; b) SRV "Deneb".

SRV "Deneb" equipment:

- SEACAT SBE19 hydrological CDT-probe;

- RCM 9LW currents registrar;

- SES-2000 light (Innomar Technologie GmbH) escaladieu parametric profilograph;

- 13.540 B gravity dirt tube with the possibility of establishing piston system (Piston/Gravity corer Model 13.540B);

- San++ automatic flow analyzer with the sampler SA1100, containing 2 x 50 sample positions;

- deep-sea sampling carousel complex;

The equipment of the vessel "Deneb" for hydrological and lithological researches:

- the Molchanov's and Niskin's samplers for sampling water;

- the Petersen's and Van-Veena's bottom samplers for sampling of bottom sediments;

- the bentonia dredge;

- the Epstein's and Jedi's plankton nets for sampling plankton;

- the caviar net for sampling ichthyoplankton;

- the set of nets and dredges for ichthyological researches;

- dirt direct flow pipe with the possibility of taking a column of precipitation $2-2.5 \mathrm{~m}$.

The hydrochemical laboratory is located on the board of the vessel "Deneb" due to which we can performed all complex of fiend analyzes (oxygen, nutrients, $\mathrm{pH}$, sulfide, plant pigments, production-destruction).
The equipment of the vessel "Deneb" for ichthyological research:

- bottom bentrawl;

- spacer pelagic trawl (28 m - in horizontal, $8 \mathrm{~m}$ - in vertical);

- the SBE19 plus hydro-probe;

- the SBE43 dissolved oxygen sensor;

- PC (personal computer) with software for hydrophysical probes;

- bathometers 31 и 51 , complete equipment for the measurement of oxygen concentration by Winkler method;

- (ADCP) profiler WHS 600.

\section{Work content}

The expeditions were carried out in the framework of the RSF project No. 17-1101286 "Predictive modeling of hydrophysics and biological kinetics processes in inland water and marine systems of the South of Russia on supercomputers", the purpose of which is to obtain systematic information of the influence of chemical and physical water properties on the ecological situation of the Azov Sea [7-14].

Oceanographic, hydrochemical, hydrobiological observations were carried out according to standard procedures and guidelines for conducting oceanographic researches with the modern expedition equipment. Complex researches of the Azov Sea and Taganrog Bay are included: meteorological, hydrological and hydrochemical observations; research of peculiarities of spatial distribution of plankton and benthos. Hydrometric and hydrological works are included: monitoring the temperature of the water, air, depth, transparency and color of the water, the excitement, the speed and direction of wind, visibility and cloudiness, velocity and oxygen concentration analysis of the species composition and concentration of planktonic populations; measurement of water temperature and selection of its sample, followed by determination of salinity using the samplers at each of the stations less than $7 \mathrm{~m}$ from two, and in depths greater than $7 \mathrm{~m}-$ from three horizons (surface, $5 \mathrm{~m}$, near the bottom). Upon detection of temperature gradients, the measurements of temperature and water sampling for salinity were performed in additional horizons.

Quality observation program of marine waters are included the following activities:

- the determination of chemical compounds concentration: petroleum hydrocarbons, $\mathrm{mg} / \mathrm{dm}^{3}(\mathrm{mg} / \mathrm{l})$; dissolved oxygen, $\mathrm{mg} / \mathrm{dm}^{3}(\mathrm{mg} / \mathrm{l}, \%) ; \mathrm{pH}$; chlorinated hydrocarbons, including pesticides, $\mathrm{mkg} / \mathrm{dm}^{3}(\mathrm{mkg} / \mathrm{l})$; heavy metals - mercury, lead, cadmium, copper, $\mathrm{mkg} / \mathrm{dm}^{3}(\mathrm{mkg} / \mathrm{l})$; phenols, $\mathrm{mkg} / \mathrm{dm}^{3}(\mathrm{mkg} / \mathrm{l})$; synthetic surface-active substances, $\mathrm{mkg} / \mathrm{dm}^{3}(\mathrm{mkg} / \mathrm{l})$;

- the determination of indicators and nutrients, specific to the selected area: the nitrite nitrogen, $\mathrm{mkg} / \mathrm{dm}^{3}(\mathrm{mkg} / \mathrm{l})$; the silicon, $\mathrm{mkg} / \mathrm{dm}^{3}(\mathrm{mkg} / \mathrm{l})$; the water salinity, $\%$; the water and air temperature, ${ }^{\circ} \mathrm{C}$; the wind speed and direction, $\mathrm{m} / \mathrm{sec}$; water transparency (color points); sea rough (points); 
- the performing visual observations of the sea water object surface.

\section{Pulsation measurements of the velocity vector of water flow}

The parameterization problem of turbulent exchange coefficient occurs in modeling the water flow fields. Turbulent exchange influence to the intensity of such processes as seabed erosion, sediment transport and transport of impurities [15-18]. The ability of water to cleanse itself depends on mechanisms of turbulent mixing.

Stochastic methods are often used for description turbulent flows. Various fluctuating quantities are considered as random functions. Turbulence has a complicated statistical structure due to the strong intermittency at the dissipative scales.

Mechanisms of vertical turbulent exchange are suppressed in numerical modeling on large vertical grids. This fact determines the necessary using small scale vertical resolutions.

Data of velocity pulsations in some points of shallow systems were obtained during the expedition with using ADCP (Acoustic Doppler Current Profiler) WHS600 Sentinel. The ADCP-profiler was fixed at free water surface. The measurements were carried out vertically from the free surface to the bottom (Fig. 2). The ADCPprofiler works on the Doppler's effect, transmitting the audio signal at a fixed frequency and receiving echoes of returned signal using the sound emitter through the entire depth of the water column. The emitter radial velocity (along-beam) is calculated from the Doppler's echo. Ripple velocity components $\left(u^{\prime}, v^{\prime}, w^{\prime}\right)$ are calculated from the values of radial velocities.

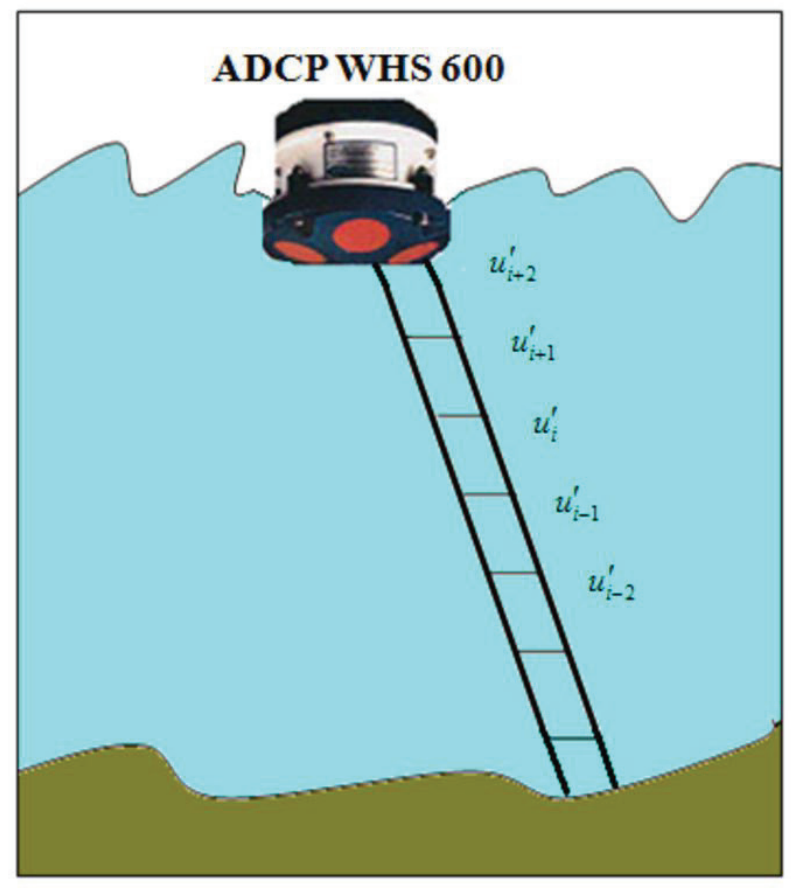

Fig. 2. ADCP-profiler position, fixed on the free water surface
The barometrically selection of samples was done, and the analysis of water composition was performed during the researches. The table, contained the measured values of dissolved oxygen concentration in the horizons of the researched water at the stations the expedition route, are shown below (Table 1).

Table 1. Dissolved oxygen concentration in the Azov Sea

\begin{tabular}{|c|c|c|c|c|c|}
\hline \multirow{2}{*}{$\begin{array}{c}\text { Statio } \\
\text { n No. }\end{array}$} & \multirow{2}{*}{$\begin{array}{c}\text { Station } \\
\text { coordinates }\end{array}$} & \multicolumn{4}{|c|}{ Measured values of dissolved } \\
oxygen
\end{tabular}

\section{The vertical turbulent viscosity coefficient}

The D. Smagorinski's model is also an algebraic model of the "mixing length", however, it is also known as subgrid model of the vertical turbulent exchange coefficient.

The principle is the following: in the case if the vertical resolution of the computational grid allows to reproduce all mechanisms of the simulation process to the scale of viscous dissipation of very small vortices, the turbulence is directly calculated from the NavierStokes equations and is naturally obtained in numerical simulations. Thus mechanisms of vertical turbulent exchange are suppressed in numerical modeling on large vertical grids. This fact determines the necessary using small scale vertical resolutions. The D. Smagorinski's idea consists in adding the turbulent viscosity to molecular viscosity. The turbulent viscosity, in turn, is determined by the mixing length, which corresponds to the size of small eddies.

Method for determine the coefficient of vertical turbulent viscosity are divided into two categories, depending on the observational data: the distribution data of average speed and data of pulsation measurements of the Reynolds stresses. The coefficient of vertical turbulent viscosity, defined by the first method, changes in interval of $0.1-10^{3} \mathrm{~cm}^{2} / \mathrm{s}$. The second method requires the use of sophisticated equipment, which can be used to measure the velocity fluctuations of water flow. The turbulent viscosity coefficient can be defined as the ratio of the Reynolds stresses to the gradient of average velocity (according to the A.S. Monin). It is known that the density stratification is virtually absent in shallow waters, and hydrodynamic processes in the vertical direction are essentially nonlinear. So we have to resort to non-standard third method of measurement and evaluation the coefficient of vertical turbulent viscosity, based on the calculation of the average velocity gradient of the water flow. 
Among the various approximations of the coefficient of vertical turbulent exchange the algebraic subgrid models are the most applicable. These models are based on the determination of turbulent flows as averaged multiplication of deviations over space or time (correlation) of the velocity components of the currents and a transferring physical quantity.

Evaluation methods of turbulence characteristics on the movement or scattering markers (indicators, buoys, etc.) are subgrid methods. The large measured data are necessary to evaluate the characteristics of vertical turbulence for direct subgrid methods. This is directly related to the duration and high cost of expidition researches.

Due to numerical simulation of turbulence we can obtained the coefficient of vertical turbulent exchange, inhomogeneous in depth, on the basis of measured velocity pulsations of water flow.

In works [19-24] it was shown that the best results, closest to experimental field data, were obtained on the basis of the O.M. Belotserkovsky parametrization.

In the case if the vertical resolution of the computational grid allows to reproduce all mechanisms of the simulation process to the scale of viscous dissipation of very small vortices, the turbulence is directly calculated from the Navier-Stokes equations and is naturally obtained in numerical simulations. Thus, the dissipation rate can be expressed through the average strain rate of cell scale in numerical modeling on large vertical grids :

$$
\bar{s}=2 \bar{s}_{i j} \cdot \bar{s}_{i j},
$$

where $\bar{S}_{i j}$ is the averaged tensor of deformation rate: $\bar{S} \sim \varepsilon^{2 / 3} \Delta^{-4 / 3}$. form:

The expression for the turbulent viscosity has the

$$
v=C_{s}^{2} \Delta^{2}{ }^{1 / 2}
$$

where $\mathrm{C}_{\mathrm{s}}$ is the dimensionless empirical constant, whose value is determined based on the calculation process of the decay of homogeneous isotropic turbulence. Particularly, the selected constant value $C_{s}$ must ensure the conformity of simulation results with experimental measurements. As a rule, the values of the $C_{s}$ constant in most cases, vary from 0.1 (in the flow channel) to 0.2 (isotropic turbulence).

In the case if the grid size is selected sufficiently small, the average rate of strain cell scale $\bar{s}$ is equaled to the average deformation rate in the Prandtl's parameterization $D$. Thus, we obtained the Prandtl's analogue in the form:

$$
v=C_{s}^{2} \Delta^{2} \sqrt{2 D_{i j} D_{i j}},
$$

Neglecting horizontal gradients $\bar{u}$ и $\bar{v}$ and gradients of the vertical velocity component $\bar{w}$, we obtained the following:

$$
v=C_{s}^{2} \Delta^{2} \frac{1}{2} \sqrt{\left(\frac{\partial \bar{u}}{\partial z}\right)^{2}+\left(\frac{\partial \bar{v}}{\partial z}\right)^{2}} .
$$

The D. Smagorinski's model of turbulent viscosity sometimes called as a theory of subgrid scale of the first order. It is easy to analyzed the algebraic models, discussed above, and this model.

\section{Numerical calculation of vertical turbulent viscosity coefficient}

The numerical experiments were performed on the basis of the above approaches for all points, $v \sim \varepsilon^{1 / 3} \cdot \Delta^{4 / 3}$, in which field measurements were conducted during the expedition researches. In the results the coefficients distribution of vertical turbulent exchange for the Azov Sea were obtained (Fig. 3).
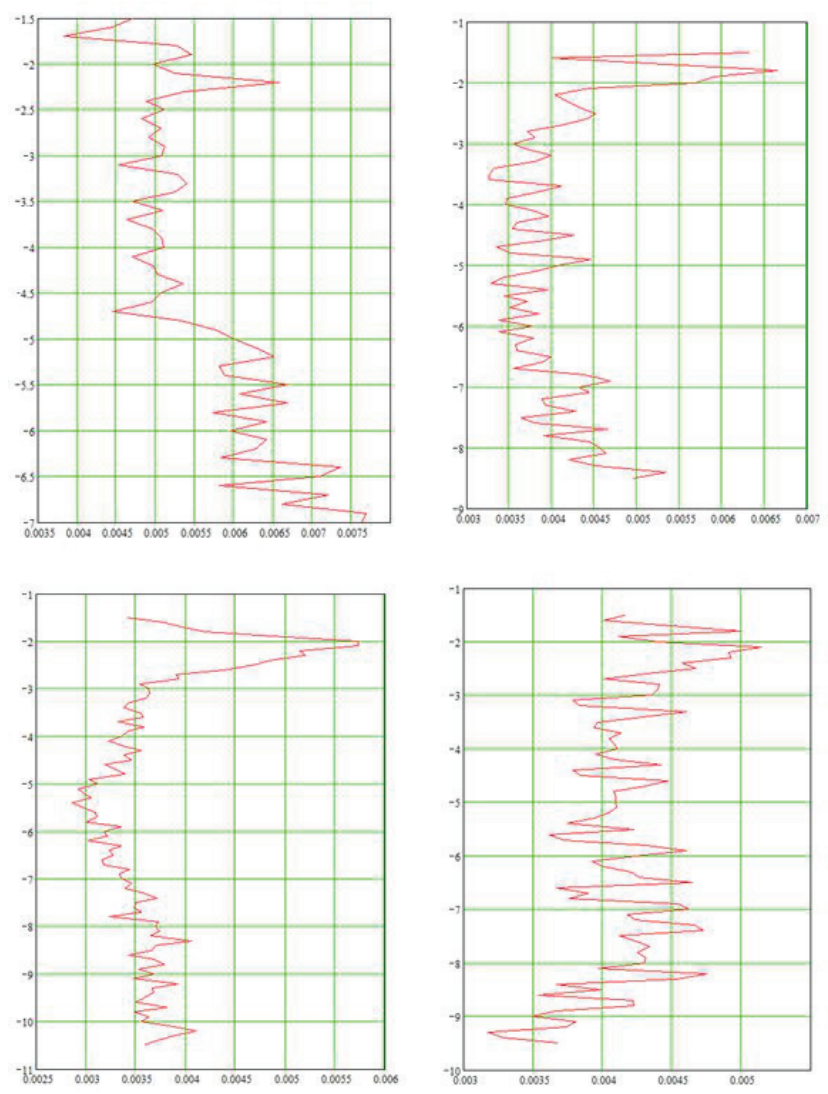

Fig. 3. The vertical turbulent exchange coefficient in points 1 , 2, 3, 12 (in horizontal - values, $\mathrm{m}^{2} / \mathrm{sec}$,

in vertical - the water level from the free surface to the bottom) (according to the D. Smagorinski).

The impact of changes in the values of vertical turbulent exchange coefficient on the concentration of dissolved oxygen in the bottom layer of a shallow water was researched. The analysis of simulation results of hydophysics processes is shown that the highest concentration of dissolved oxygen in the bottom layer of the Azov Sea is corresponded to the highest value of vertical turbulent exchange coefficient (point 12), and the lowest value of vertical turbulent exchange coefficient is corresponded to the lowest value of dissolved oxygen concentration (point 3 ). The values of vertical turbulent exchange coefficient is close to zero at the depth of about 3 meters and below. This means that the turbulent exchange is reduced in the vertical in this region, and explains the 
phenomenon of anoxia in the bottom layer of the Central-Eastern part of the Azov Sea.

\section{Conclusion}

The concentrations of dissolved oxygen and data of velocity pulsations of water flow in some points of shallow systems were obtained during the expedition researches with using ADCP WHS600 Sentinel. Areas with low dissolved oxygen content were discovered in the bottom water layer.

The distributions of vertical turbulent exchange coefficient with data of velocity component pulsations, measured during the expedition, were obtained experimentally.

The conformity of calculation results based on the statistical velocity field data of water flow and the subgrid Smagorinski's models with field measurements was obtained.

The impact of changes in the values of vertical turbulent exchange coefficient on the concentration of dissolved oxygen in the bottom layer of the shallow water was researched.

This paper was partially supported by the grant No. 17-1101286 of the Russian Science Foundation.

\section{References}

1. B.N. Chetverushkin, Mathematical Models and Computer Simulations, 5, No. 3, 266-279 (2013)

2. A.I. Sukhinov, E.A. Protsenko, A.E. Chistyakov, S.A. Shreter, Computational Methods and Programming. 16, No. 3., 328-338 (2015)

3. A. I. Sukhinov, A. E. Chistyakov, E. F. Timofeeva, A. V. Shishenya, Mathematical Models and Computer Simulations. 5, No. 2., 122-129 (2013)

4. A.I. Sukhinov, A.E. Chistyakov, Computational Methods and Programming, 13, No. 1, 290-297 (2012)

5. A.I. Sukhinov, A.E. Chistyakov, A.A. Semenyakina, A.V. Nikitina Computational Methods and Programming. 16, No. 2., 256-267 (2015)

6. O. M. Belotserkovskii, Turbulence: New Approaches (Nauka, Moscow, 2003).

7. Samarskii A.A.The theory of difference schemes (Science, Moscow, 1989)

8. O.M. Belocerkovskij, V.A. Gushhin, V.V. Shhennikov, USSR Computational Mathematics and Mathematical Physics, 15, No. 1, 190-200 (1975)

9. I.Y. Gluhenky, A.V. Lavrent'ev, G.G. Popov, Safety in Technosphere, No. 6., 3-6 (2011)

10. A.S. Abdusamadov, A.P. Panarin, A.K.Magomedov L.D. Kovalenko, B.R. Huseynov, A.M. Dohtukaeva, L.A. Dudurhanova, Geography and Geoecology. Southern Russia: ecology, razvitie., №1., 165-166 (2012).

11. Resolution of the Government of the Russian Federation on April 15, 2002 № 240 "On the procedure for organizing the activities for prevention and liquidation of oil spills in the Russian Federation "

12. https://www.gismeteo.ru/diary/5211/2007/11/

13. A.I. Sukhinov, A.E. Chistyakov, E.A. Protsenko, Mathematical Models and Computer Simulations, 6, No. 4, 351-363 (2014)

14. A.I. Sukhinov, A.E. Chistyakov, E.A. Protsenko, Computational Methods and Programming., 15, 610-620 (2014)

15. A.I. Sukhinov, A.V. Nikitina, A.E. Chistyakov, Mathematical Models, 24, No. 9., 3-21 (2012)

16. A.V. Nikitina, A.I. Sukhinov, G.A. Ugolnitsky, A.B. Usov, A.E. Chistyakov, M.V. Puchkin, I.S. Semenov, Mathematical Models and Computer Simulations, 9 (1), 101-107 (2017)

17. A.I. Sukhinov, A.E. Chistyakov, I.I. Levin, I.S. Semenov, A.V. Nikitina, A.A. Semenyakina, 5th ICIEV , 1128 - 1133 (2016)

18. A.I. Sukhinov, A.E. Chistyakov, A.V. Nikitina, A.A. Semenyakina, I. Korovin, G. Schaefer, 5th ICIEV, 1134 - 1139 (2016).

19. A.I. Sukhinov, A.E. Chistyakov, A.A. Semenyakina, A.V. Nikitina, Computer researches and modeling. 8, no. 1., 151 - 168 (2016)

20. A.I. Sukhinov, D.S. Khachunts, A.E. Chistyakov, Computational Mathematics and Mathematical Physics. 55, No. 7, 1216-1231 (2015)

21. A.I. Sukhinov, A.E. Chistyakov, A.V. Shishenya, Mathematical Models and Computer Simulations, 6, No. 3., 324-331 (2014)

22. N. Buzalo, P. Ermachenko, T. Bock, A. Bulgakov, A. Chistyakov, A. Sukhinov, E. Zhmenya, N. Zakharchenko, Proc. Eng., 85, 84-93 (2014)

23. A.I. Sukhinov, A.E. Chistyakov, E.F. Timofeeva, A.V. Shishenya, Mathematical Models and Computer Simulations, 5, No. 2., 122-129 (2013)

24. A.I. Sukhinov, A.E. Chistyakov, Mathematical Models and Computer Simulations, 4, Iss. 4, 398409 (2012). 